\title{
Conocimiento de la educación y función pedagógica: el sentido de la competencia profesional
}

JosÉ MANUEL TOURIÑÁN LÓPEZ

Universidad de Santiago de Compostela

\footnotetext{
SUMMARY.-This article carrys out the axe of discussion about the development of education as a subject of knowledge.

In fact, the content focusses three basic questions:

a) The distintion between cultural matters and education as a subject of knowledge. (Knowing disciplines and knowing education).

b) Arguments about educational knowledge such as specialized one.

c) Teaching centres for Educational Specialists.
}

\section{INTRODUCCIÓN}

Tomando como eje argumental el desarrollo del conocimiento de la educación, es decir, no los conocimientos de las áreas culturales que se enseñan, ni el conocimiento como objeto de educación, sino la educación como objeto de estudio y conocimiento, este trabajo pretende defender el carácter especializado del conocimiento de la educación, y, como consecuencia, la necesidad de que ese conocimiento especializado se tenga en cuenta al establecer la competencia profesional de los especialistas en funciones pedagógicas.

Para desarrollar estas ideas, dividiremos la exposición en tres partes:

- Diferencias entre el «conocimiento de áreas culturales» y el «conocimiento de la educación».

- Objeciones al carácter especializado del conocimiento de la educación.

- Los centros de formación de especialistas en funciones pedagógicas.

\section{CONOCIMIENTO DE ÁREAS CULTURALES Y CONOCIMIENTO DE LA EDUCACIÓN}

Los trabajos que se realizaron en el curso de doctorado que dirigí en el Departamento de Pedagogía Sistemática durante 1985 pusieron de manifiesto una fuerte tendencia a considerar de manera secundaria los conocimientos pedagógicos.

En ese curso monográfico se intentaba dar respuestas a la siguiente pregunta: ¿qué tiene que saber un profesional de la docencia que no adquiera ya por el hecho 
de dominar el área cultural en la que va a impartir docencia? Por supuesto, es evidente que la función pedagógica no se reduce a la docencia; el colectivo profesional de los docentes es sólo una parte de los profesionales de la educación. Pero ello no anula la pertinencia de la pregunta para ver si, en efecto, existe una consideración secundaria del conocimiento de la educación.

Antes de continuar con esta reflexión, conviene precisar dos conceptos:

A) En el sistema educativo trabajan sociólogos, médicos, psicólogos, y otros profesionales que reciben con propiedad la denominación de profesionales del sistema educativo. Pero, además, existe un grupo de profesionales del sistema educativo que merecen con propiedad la denominación de profesionales de la educación; su tarea es intervenir, realizando las funciones pedagógicas para las que se han habilitado. En mi opinión, «profesionales del sistema educativo» y «profesionales de la educación» son dos expresiones distintas con significado diferente; y tiene sentido afirmar que, no todo profesional del sistema educativo es profesional de la educación, en tanto en cuanto sólo el contenido de la formación profesional de este es siempre el conocimiento de la educación. Profesional de la educación es el especialista que domina los conocimientos teóricos, tecnológicos y prácticos de la educación que le permiten explicar, interpretar y decidir la intervención pedagógica propia de la función para la que se está habilitando.

B) Si tomamos como referente las tareas y actividades a realizar en el ámbito educativo, el conocimiento de la educación y el crecimiento del sistema educativo permiten identificar tres tipos de funciones pedagógicas genéricamente:

- Funciones de docencia, identificadas básicamente con el ejercicio y dominio de destrezas, hábitos, actitudes y conocimientos que capacitan para enseñar en un determinado nivel del sistema educativo.

- Funciones de apoyo al sistema educativo. Son funciones que no se ocupan directamente de la docencia, aunque mejoren las posibilidades de ésta, porque su tarea es resolver problemas pedagógicos del sistema educativo que surgen con el crecimiento del mismo y del conocimiento de la educación, y que, de no subsanarse, paralizarían la docencia o dificultarían el logro social de una educación de calidad a través del sistema educativo, como es el caso de la organización escolar, la intervención pedagógico-social, la planificación educativa, etc.

- Funciones de investigación pedagógica, identificadas con el ejercicio y dominio de destrezas, hábitos, actitudes y conocimientos que capacitan para la validación y desarrollo de modelos de explicación, interpretación y transformación de intervenciones pedagógicas y acontecimientos educativos.

Retomando de nuevo nuestro tema, puede decirse que, cuando a la pregunta formulada anteriormente se responde de manera genérica que, por ejemplo, los licenciados en Historia están más preparados que los profesores de E.G.B. para explicar un tema de Historia a los escolares del ciclo superior de la E.G.B., porque tienen mayor dominio del contenido cultural del tema a explicar, no cabe duda de que se está manifestando una apreciación secundaria de los conocimientos pedagógicos, porque cualquier diplomado de E.G.B. de la especialidad de sociales dispone, por formación, no sólo de los contenidos culturales del tema suficientes para el estudiante de E.G.B., sino que además dispone de un bagaje pedagógico que le coloca 
en situación ventajosa respecto al licenciado en Historia para conseguir explicar el tema en cuestión.

Al margen de las casuísticas particulares, me parece a mí que respuestas negativas como la anterior, sólo tienen sentido si se acepta, o bien que la «explicación didáctica» no es un concepto con significación intrínseca distinto de «explicación científica», sino cuestión de práctica, o bien que el conocimiento de la función pedagógica que se ha transmitido en los centros de formación de especialistas no es eficaz.

En este sentido, y tomando como ejemplo la función de docencia, la Comisión que ha confeccionado el «Documento de bases para la elaboración del estatuto del profesorado» mantiene que el núcleo pedagógico formativo del profesor debe desarrollar en éste:

«La capacidad de diagnóstico del individuo y del grupo de aprendizaje; de concreción y acomodación de los objetivos y programas; de formulación y experimentación de procedimientos y estrategias metodológicas; de organización del espacio, del tiempo de las relaciones en el aula; de diseño de procedimientos, técnica e instrumentos de evaluación del aprendizaje y de los mismos procesos de enseñanza; de interpretación de resultados y de reformulación consecuente de los planteamientos de partida» (Comisión..., 1986, p. 10).

El nivel de las investigaciones pedagógicas actuales permite afirmar que hay razones suficientes para distinguir y no confundir en el lenguaje técnico:

- el conocimiento de la educación, y

- los conocimientos de las áreas culturales.

Es verdad que desde el punto de vista antropológico, la educación es cultura, y, por tanto, tiene sentido afirmar que la función del profesional de la educación es transmitir cultura. Pero, si además afirmamos que los términos educacionales carecen de contenido propio, los conocimientos de las diversas áreas culturales se convierten en el eje de toda actividad pedagógica hasta el extremo de que los mismos profesionales de la educación llegan a aceptar que su formación es simplemente el conocimiento de esas áreas culturales.

El análisis detenido del contexto pedagógico da pie para sostener que el conocimiento de las áreas culturales no es el conocimiento de la educación, porque:

a) Si bien es verdad que una buena parte de los objetivos de la educación tiene algo que ver con los contenidos de las áreas culturales, el ámbito de los objetivos no se agota en los ámbitos de las áreas culturales. La función pedagógica no se agota en saber qué nivel de información cultural se está consiguiendo al desarrollar un tema de un área cultural en una clase; antes bien, la función pedagógica se pone de manifiesto cuando se sabe qué tipo de destrezas, hábitos, actitudes, etc., de los diversos dominios que señalan las taxonomías se están potenciando al trabajar de manera especial en ese tema. La cuestión no es saber tanta Historia como el historiador, sino saber qué objetivos de conocimiento se logran y cómo se logran al enseñar un tema de Historia y qué destrezas, hábitos, actitudes, etc., estamos desarrollando al enseñar ese tema.

b) La identificación del conocimiento de las áreas culturales con el conocimiento de la educación fomenta una situación pedagógica insostenible: la tendencia 
a evaluar el rendimiento escolar fundamentalmente por los niveles de información cultural. Sin que ello signifique que cualquier contenido sea puramente formal y sirva para alcanzar cualquier tipo de destreza, es posible afirmar que, aunque no con el mismo nivel de eficacia, desde el punto de vista pedagógico, con uno sólo de los temas culturales del programa que debe estudiar un alumno de Ciclo Medio, por ejemplo, se podrían poner en marcha las estrategias pedagógicas conducentes al logro de casi todos los objetivos educativos del programa, a excepción de la información cultural.

c) Incluso identificando conocimiento de la educación y conocimiento de áreas culturales, se puede entender que hay un determinado conocimiento de la educación que no es el conocimiento de las áreas culturales: el conocimiento de la transmisión de esos conocimientos de esas áreas culturales.

La Educación tendría efectivamente como misión, por ejemplo, la transmisión del conocimiento histórico. Que ese conocimiento histórico sea fiable y válido es problema de los historiadores; el conocimiento de la educación sería el de las estrategias de intervención.

Los conocimientos teóricos, tecnológicos y práxicos que se constituyen en objetivos de conocimiento de la enseñanza, no los crea el profesional de la educación. Son los investigadores de cada área cultural los que crean. Al profesional de la educación le corresponde con fundamento de elección técnica decidir: si el educando puede aprenderlos, si son coherentes con la representación conceptual de la intervención, si tienen fundamento teóricos, tecnológico y práxico, según el caso, cual es el método de enseñanza adecuado y que destrezas, hábitos y actitudes se pueden desarrollar con la enseñanza de ese conocimiento. Es decir, el profesional de la educación domina los conocimientos teóricos, tecnológicos y práxicos del área cultural que va a enseñar. Pero como profesional de la educación domina el conocimiento de la educación que le permite justificar y explicar la conversión de esos conocimientos de un área cultural en objetivo o instrumento de la intervención pedagógica.

El conocimiento de la educación capacita al profesional de la docencia, no sólo para establecer el valor educativo de un contenido cultural y participar en el proceso de decidir su conversión en fin o meta de un determinado nivel educativo, sino también para establecer programas de intervención ajustados a hechos y decisiones pedagógicas que hagan efectiva la meta propuesta.

Hablar del conocimiento de la educación no implica, por tanto, interrogarse directamente acerca de los saberes de las áreas culturales. Cuando hablamos de «el conocimiento de la educación» es más apropiado preguntarse por qué determinados conocimientos se constituye en meta e instrumento de la acción educativa o por qué es educable la dimensión cognitiva del hombre. Y así como de los conocimientos de cada área cultural podrían hablarnos, según el caso y con propiedad, el historiador, el geógrafo, el matemático, el físico, etc., porque son especialistas de cada una de esas áreas de conocimiento, no me cabe ninguna duda que responder adecuadamente a si tal o cual contenido histórico, matemático, físico, etc., debe constituirse en el contenido de la acción educativa que realizamos con un determinado sujeto, o a cómo cultivar su sentido crítico, exige interrogarse acerca de la educación como objeto de conocimiento. En el primer supuesto, los conocimientos de áreas culturales 
- la historia, la matemática, la física, etc.— son el objeto científico de estudio; en los dos casos del segundo supuesto, la transmisión misma, la influencia que se ejerce, se convierte en objeto específico de la reflexión científica.

De acuerdo con las reflexiones realizadas anteriormente, hablar de «conocimiento de la educación» es lo mismo que interrogarse acerca de la educación como objeto de conocimiento, lo que equivale a formular una doble pregunta:

a) Qué es lo que hay que conocer para entender y dominar el ámbito de la educación, o lo que es lo mismo, cuales son los componentes del fenómeno educativo que hay que dominar para entender dicho fenómeno.

b) Cómo se conoce ese campo, o dicho de otro modo, que garantías de credibilidad tiene el conocimiento que podamos obtener acerca del campo de la educación.

Me parece necesario distinguir conocimiento de áreas culturales y conocimiento de la educación porque, en la misma medida que el conocimiento de la educación va más allá de lo que se transmite, la función pedagógica comienza a ser objeto de conocimiento especializado y específico.

Si no distinguimos conocimiento de áreas culturales y conocimiento de la educación, se sigue que, por ejemplo, la competencia profesional de los profesores se definiría erróneamente por el mayor o menor dominio del área cultural que van a enseñar. Este tipo de planteamientos genera consecuencias nefastas para estos profesionales:

a) En primer lugar, como los conocimientos de áreas culturales que enseñan no los crearían los profesores, estos se percibirían a sí mismos como aprendices de los conocimientos de esas áreas que otros investigan.

b) En segundo lugar, como la competencia profesional se definiría por el dominio del área cultural, se fomentaría el error de creer que el que más sabe es el que mejor enseña.

Si no confundimos conocimiento de áreas culturales y conocimiento de la educación, ni es verdad que el profesor es un aprendiz de las áreas culturales que enseña, ni es verdad que necesariamente el que más Historia sabe es el que mejor la enseña, ni es verdad que el que mejor domine una destreza es el que mejor enseña a otro a dominarla, a menos que, tautológicamente, digamos que la destreza que domina es la de enseñar.

Esto es así, porque cada una de esas actividades requiere distintas competencias y destrezas para su dominio, y la pericia y perfección en una de ellas no genera automáticamente el dominio de la otra.

En rigor lógico, hay que aceptar que el conocimiento de la educación es, pues, un conocimiento especializado que permite explicar, interpretar y decidir la intervención pedagógica propia de la función para la que se habilita, bien sea función de docencia, bien sea de apoyo al sistema educativo, o bien sea función de investigación.

\section{OBJECIONES A LA NECESIDAD DE CONOCIMIENTO ESPECIALIZADO}

Si repasamos las afirmaciones anteriores, parece obvio que la función pedagógica, por principio de significado, exige conocimiento especializado de la educación. 
Se requiere conocimiento especializado, porque en el transcurso de los dos últimos siglos se ha acumulado un núcleo científico de conocimiento acerca de la educación, sin el cual el control de la intervención pedagógica, su conocimiento y justificación no es posible.

Además del dominio adecuado de los conocimientos del área cultural en la que se imparte docencia - tomando como referencia una de las funciones pedagógicas-, el control del proceso educativo, en este caso, supone no sólo el conocimiento de la estructura cognitiva del sujeto que aprende y el espacio sociocultural en el que aprende -como los estudios interdisciplinares de la educación han probado-, sino también el conocimiento de un cuerpo teórico que justifique la formulación de normas de intervención pedagógica.

Con todo conviene resumir las objeciones que se han hecho a la necesidad de conocimiento especializado para la función pedagógica.

Una primera objeción mantiene que el conocimiento especializado no es condición necesaria en la función pedagógica porque, si bien es verdad que en la formación de especialistas existe un núcleo pedagógico, también es verdad que hay educación en la que no intervienen especialistas.

Es innegable que los padres educan; es innegable que hay procesos de educación informal, que hay autoeducación, e incluso, como dicen algunos, educación espontánea. Sin embargo, no puede considerarse seriamente esta objeción.

Que el conocimiento especializado sea necesario, no significa que cualquier tipo de intervención educativa requiera el mismo nivel de competencia técnica.

En mi opinión, estos ejemplos no prueban que el conocimiento especializado no sea necesario, sino que todo tipo de intervención educativa no requiere el mismo nivel de competencia pedagógica. Un padre de familia, no experto en Pedagogía, sabe que, obrando de un modo especial —que ha visto o que han utilizado con él一, se consigue un cierto efecto educativo. Pero el conocimiento de las razones por las cuales obrando de ese modo se consigue ese efecto, es una competencia teórica que requiere estudio especializado. Sólo en la medida que dominamos esa competencia, estamos en condiciones de controlar el proceso y mejorar la intervención. Es necesario reconocer que no toda intervención educativa es, técnicamente hablando, una intervención pedagógica.

La función pedagógica requiere conocimiento especializado, pero la realización de una acción educativa no exige más nivel especializado de competencia técnica que el requerido para hacer efectiva la meta propuesta. $Y$ esto quiere decir que existen muy diversas intervenciones que no pueden ser resueltas sin alto nivel de competencia técnica y que existen otras intervenciones cuya generalización y repetición las convierten en conocimientos especializados de uso común.

Aunque no con el grado de elaboración que tiene en las acciones de los profesionales de la educación, el conocimiento especializado está presente en la función pedagógica, personal, familiar e informal.

Por otra parte, debe aclararse que la educación espontánea no es argumento en contra de la necesidad de conocimiento especializado en la función pedagógica.

Existe, por supuesto, educación espontánea, es decir, obtención de resultados educativos en actividades que no están conformadas como estímulos directamente o 
no directamente educativos. Es el caso en que un niño juega a las carreras y sin que su intención sea otra de jugar, está desarrollando destrezas motóricas de indudable interés educativo.

Sencillamente, en la educación espontánea no hay función pedagógica. Pero sirve para probar la necesidad de conocimiento especializado, porque gracias a él somos capaces de introducir en procesos controlados ese desarrollo espontáneo de destrezas educativas.

Otra objeción bastante generalizada mantiene que la función pedagógica no necesita el conocimiento especializado que proporciona la Pedagogía, entendida como conocimiento científico autónomo de la educación, tal como si la experiencia de la propia práctica proporcionase el conocimiento de la educación necesario. Se argumenta en este caso que existen profesionales que realizan su función de manera eficaz sin recibir formación en el núcleo pedagógico. Es su dominio del contenido de la materia que enseñan y la experiencia personal de su actuación lo que les convierte en grandes profesores.

De manera formal se nos dice que, si la función pedagógica requiere más conocimiento especializado que el de la propia práctica, no se podría explicar por qué durante siglos la transmisión de cultura se logró sin conocimiento científico de la función pedagógica.

Es un hecho innegable que históricamente la función pedagógica fue considerada como una actividad puramente práctica; no era objeto de conocimiento científico; el acto de intervención era estudiado experiencialmente. No habría ciencia de la función pedagógica, pero la experiencia proporcionaba el conocimiento especializado de la función.

Al criticar esta objeción no se trata de negar el valor de la experiencia, se trata más bien de poner de manifiesto que en los productos culturales - y la función pedagógica lo es - la ausencia de una determinada condición para obtener un resultado en una época histórica concreta, no es prueba de que no sea necesaria la presencia de esa condición en otra época para obtener un resultado, a menos que defendamos que el resultado a obtener sea el mismo y no han variado, ni los conocimientos de las personas sobre ese ámbito, ni los modos de ordenar las acciones para lograrlo.

Dejando al margen el hecho de que no toda función pedagógica es función de docencia, es perfectamente legítimo suponer que el historiador sabe historia, el físico sabe física, y que por el hecho de saber esos contenidos, saben cómo ordenarlos a fin de producir las verdades de esas áreas de conocimiento que, cuando se repiten al oyente con la suficiente frecuencia y el ritmo adecuado, son aprendidas. Cuando el objetivo es la presentación de conocimientos de áreas culturales, es indiscutible que la claridad, el entusiasmo, la sinceridad, la flexibilidad y el dominio de la materia a enseñar, configuran el bagaje experiencial de la enseñanza.

Se explica por esta razón que fundamentalmente a nivel universitario un profesor necesite «menos pedagogía» para que sus alumnos aprendan; lo normal es que los alumnos universitarios dispongan de las destrezas educativas adecuadas para introducirse en contenidos culturales de alto nivel de abstracción. Si, además, no nos olvidamos de que los alumnos universitarios son especialistas — después de muchos 
años de práctica - en descifrar mensajes verbales, hemos reunido las condiciones que explican por qué hay grandes maestros en la Universidad sin estudios científicos de la función pedagógica. Si eso no fuese así, sería impensable la transmisión de la cultural de las generaciones adultas durante siglos. Ahora bien, esta argumentación resulta insuficiente cuando las trasladamos al nivel de pre-escolar, ya que en ese nivel los conocimientos de áreas culturales que se enseñan son escasos y las destrezas de los alumnos están sin desarrollar.

Como ya hemos visto, la virtualidad del conocimiento científico es proporcionar pautas de análisis intersubjetivables de los esquemas de acción que están implícitos en el modo de intervenir, de tal manera que podamos introducir en esquemas controlados de acción el desarrollo espontáneo o casual de destrezas.

Si no confundimos conocimiento de áreas culturales y conocimiento de la educación, la función pedagógica no equivale a saber tanta historia como el historiador, sino saber qué objetivos se logran al enseñar este tema de historia; qué destrezas, hábitos, actitudes y conocimientos debe poseer el alumno para aprender ese tema; cuáles de esas destrezas, hábitos o actitudes se refuerzan con el aprendizaje del tema y qué razones hay para utilizar ese tema y no otro como instrumento pedagógico.

La función pedagógica se pone de manifiesto especialmente en los alumnos de cualquier nivel que no alcanzan rendimiento deseable, o cuando hay que poner en marcha secuencias racionales de acción nuevas para recuperar a esos alumnos o cuando nos enfrentamos a un problema de organización escolar o a la enseñanza explícita de los principios que justifican nuestra forma de actuación. No es suficiente decir lo hago así porque es lo que exige esta materia o porque lo he hecho siempre así o porque es bueno moralmente.

La destreza en facilitar el aprendizaje, la competencia requerida para elaborar programas de recuperación de aprendizaje más allá de la mera repetición de la explicación o del estudio, la preparación para distinguir entre objetivos de conocimiento y objetivos educativos, son aspectos de la función profesional que requieren una alta elaboración teórica, imposible de alcanzar prácticamente, sin recibir una formación específica de Pedagogía.

Por último, y abundando en el argumento de la objeción anterior, algunos entienden que pueden formular una tercera objeción. Los partidarios de ella mantienen que: el conocimiento especializado no es una condición necesaria en la función pedagógica, porque la tarea educativa se ejerció en otras épocas; se ejerce, incluso hoy, sin pedagogos.

Frente a esta objeción puede afirmarse, como en el caso anterior, que, en los productos culturales, la ausencia de una determinada condición para obtener un resultado en una época histórica concreta, no es prueba de que no sea necesaria la presencia de esa condición en otra época para obtener un resultado, a menos que defendamos que el resultado a obtener sea el mismo, y no han variado ni los conocimientos de las personas sobre ese ámbito ni los modos de ordenar las acciones para lograrlo.

Si entendemos que «pedagogos» son todos aquellos profesionales cuya formación se cursa en un centro especializado que los habilita como licenciados en Ciencias de la Educación, se comprende por qué la ausencia de pedagogos equivale a la 
no necesidad de conocimiento especializado para la función pedagógica. Pero estas afirmaciones pierden significación ante las siguientes consideraciones:

1) No siempre hubo pedagogos de nombre. Sin embargo, eso no es incompatible con la existencia de la preocupación por la función pedagógica.

Unicamente cuando una función es ejercida colectivamente en un gremio comienza a tener sentido el apelativo específico del gremio. Son dos cosas distintas la preocupación por la función pedagógica y la conversión de esa preocupación en ocupación específica de un gremio.

Precisamente por esto se entiende que pudiera haber función pedagógica sin «pedagogo» (licenciados).

2) Es la existencia de una investigación científica de la educación y la necesidad de dominar unas competencias específicas para esa función lo que fundamenta la existencia de pedagogos en sentido amplio.

Hoy, mejor que en otras épocas, podemos entender - dado el carácter formalizado de las carreras de Pedagogía y del Magisterio- que son dos cosas distintas la preocupación intelectual por un ámbito y el ejercicio de la función profesional de ese ámbito. La preocupación intelectual no es exclusiva de nadie, y, por la misma razón, cabe la posibilidad de saber acerca de un ámbito sin estudiar la carrera específica de ese ámbito.

A la luz de estos datos, y a fin de evitar la neutralización de diferencias que una terminología menos precisa produciría entre función pedagógica y niveles profesionales, debería mantenerse estipulativamente que pedagogo es todo aquel en cuya formación existe un núcleo pedagógico, es decir, un conjunto de conocimientos especializados de la educación que le proporcionan las competencias propias de la intervención pedagógica correspondiente a la función para la que se habilita. Y así las cosas, si pedagogo es todo aquel en cuya formación hay un núcleo pedagógico, se sigue que el pedagogo no es sólo el especialista en funciones pedagógicas, licenciado en Ciencias de la Educación.

En cualquier caso, esta tercera objeción nos permite insistir nuevamente en una tesis básica del desarrollo del conocimiento de la educación. Hoy estamos en condiciones de defender que la preocupación pedagógica ha existido siempre, aunque no fue siempre científica; la ocupación pedagógica también ha existido siempre, aunque no fuese profesionalizada; lo que no ha existido siempre es la misma consideración para la función pedagógica, porque no siempre se le ha atribuido la misma capacidad de resolución de problemas al conocimiento de la educación.

La defensa del carácter especializado del conocimiento de la educación, permite afirmar que la función pedagógica es, en nuestros días, una actividad reconocida socialmente para cubrir necesidades sociales determinadas, una actividad específica con fundamento en el conocimiento especializado de la educación, que permite establecer y generar hechos y decisiones pedagógicas con el conocimiento autónomo de la educación. La competencia de los especialistas en funciones pedagógicas procede, por tanto, del conocimiento de la educación. 


\section{LOS CENTROS DE FORMACIÓN DE LOS ESPECIALISTAS EN FUNCIONES PEDAGÓGICAS}

Personalmente he dedicado otros trabajos a la defensa de la formación de especialistas en funciones pedagógicas y mi tesis fundamental es la siguiente: la función pedagógica es una actividad específica, fundaba en conocimiento especializado, porque es posible establecer hechos y generar decisiones pedagógicas con conocimiento autónomo de la educación, y debe ser reconocida socialmente, porque es necesaria para satisfacer la necesidad social de calidad de educación. Desde estos presupuestos defiendo la identidad profesional del pedagogo y la diversidad profesional de los especialistas en funciones pedagógicas. Estos presupuestos favorecen el establecimiento de relaciones que permiten juzgar diversas alternativas institucionales de formación de los especialistas en funciones pedagógicas:

En mi opinión, lo propio del profesional de la educación es la intervención pedagógica correspondiente a la función pedagógica para la que se ha habilitado. Ahora bien, como del conocimiento de la educación que se tenga depende, no sólo la credibilidad de la función, sino también la estimación de la función por sí misma se sigue que el reconocimiento social de la función, en general, está ligado indiscutiblemente a la capacidad de resolución de problemas que se le atribuye al conocimiento de la educación.

El reconocimiento social de la función pedagógica no proviene de la estimación social del ámbito en el que se ejerce la función (en este caso la educación) sino de la competencia para resolver los problemas que se plantean en el ámbito, y por tanto, del conocimiento de la educación y de su elaboración técnica.

Frente a una profesionalización radical e irracional de la educación, la profesionalización del sistema debe entenderse más exactamente como la afirmación de que las actividades educativas pueden ser realizadas de un modo tal que cumplan las condiciones necesarias para que se considere a los que las realizan de ese modo como profesionales. Precisamente por eso se puede afirmar que los profesionales de la educación ocupan un espacio educacional definido compatible con la actuación de otros profesionales del sistema educativo y con la de otros agentes de la educación. Así las cosas, tan cierto es defender que no toda actividad educativa tiene que ser profesionalizada, como mantener que no todo tipo de intervención requiere el mismo nivel de competencia.

En mi opinión, «profesionales del sistema educativo» y «profesionales de la educación» son dos expresiones distintas con significado diferente; y tiene sentido afirmar que no todo profesional del sistema educativo es profesional de la educación, en tanto en cuanto sólo el contenido de la formación profesional de éste es siempre el conocimiento de la educación. Y así las cosas, el sentido de la perspectiva del problema no puede hacernos olvidar que sólo entre los profesionales de la educación se dan intereses y objetivos comunes respecto de su formación. En cualquier caso, el espacio de los profesionales de la educación y su formación es una cuestión ardua y pendiente todavía, en buena medida de legislación. No olvidemos las polémicas actuales acerca de los planes de estudios de Pedagogía y de la denominación de sus centros; o la polémica acerca del carácter profesional de la función directiva o de la función inspectora. 
Desde la perspectiva de profesionalización, el pedagogo se identifica lógicamente como un especialista que domina los conocimientos teóricos, tecnológicos y prácticos de la educación que le permiten explicar, interpretar y decidir la intervención pedagógica propia de la función para la que se habilita. El pedagogo es, por tanto, toda persona en cuya formación profesional hay un núcleo de conocimiento pedagógico específico para las funciones que va a ejercer. Como ya hemos dicho antes, para evitar la neutralización de diferencias que una terminología menos precisa produciría entre función pedagógica y niveles profesionales, debería mantenerse estipulativamente que pedagogo es todo aquél en cuya formación profesional hay un núcleo pedagógico, y, por consiguiente, no debe reducirse el término pedagogo al especialista en funciones pedagógicas que es licenciado en CC. de la educación.

Si tomamos como referente las tareas y actividades a realizar en el ámbito educativo, podemos afirmar que el crecimiento simple del sistema escolar y el crecimiento del conocimiento del fenómeno educativo permiten identificar hoy tres tipos de función pedagógica: funciones de docencia, funciones de apoyo al sistema educativo y funciones de investigación pedagógica. Entre sí son funciones pedagógicas distintas. Son funciones pedagógicas porque la realización de las tareas propias de cada función requiere competencias adquiridas con el conocimiento de la educación. Son funciones distintas, porque desde el punto de vista de la comprensión lógica a cada tipo de función le corresponden unas características que no se le pueden quitar sin que pierda su sentido la función; son funciones distintas, porque las tareas a realizar en cada caso tienen unas características exclusivas y requieren competencias pedagógicas diferentes.

Incluso pensando en la misma función pedagógica cabe afirmar la diversidad, porque el núcleo pedagógico puede variar cuando la capacidad de diversificación del ámbito en el que se ejerce la función justifica desde el punto de vista lógico la diferenciación en el núcleo. Tal es el caso de la función de docencia cuyo núcleo pedagógico de formación no es exactamente igual para profesores de preescolar, de segunda etapa de E.G.B., de B.U.P. o de Universidad. El núcleo pedagógico de formación puede variar dentro de la misma función, pues siendo verdad que como docentes han de dominar los conocimientos teóricos, tecnológicos y prácticos que les capacitan para explicar, interpretar y decidir la intervención pedagógica propia de su función, también es verdad que hay conocimientos pedagógicos específicos del profesor de preescolar que no mejoran en nada la capacitación profesional del profesor de universidad, como pueden ser los conocimientos pedagógicos necesarios para el desarrollo de las destrezas básicas de lectura, escritura, cálculo y expresión verbal, que son imprescindibles en la capacitación pedagógica de aquél y superfluos en la de éste.

Por la misma posibilidad lógica de diversificar el ámbito en el que se ejerce la función, puede decirse que el pedagogo social y el director escolar realizan funciones de apoyo. Sin embargo, las competencias pedagógicas requeridas para ejercer una u otra función de apoyo no son exactamente las mismas y, por consiguiente, el núcleo pedagógico, se diversifica también dentro de las funciones pedagógicas de apoyo.

De lo que acabo de exponer no se sigue que «función pedagógica» y «profesión» sean lo mismo. Es factible, en estos momentos del discurso, afirmar no sólo 
que una persona puede prepararse en diversas funciones pedagógicas, sin que ello no anule la fuerza de la distinción lógica entre ellas, sino también que, de acuerdo con criterios pragmáticos, una sola habilitación profesional puede capacitar para diversas funciones pedagógicas. Prueba de ello es, como todos sabemos, que la profesión de profesor no se identifica exclusivamente con la función de docencia aunque ésta sea su función más significativa.

Sin embargo, de lo que acabo de decir, sí se sigue la conveniencia de la Facultad de Pedagogía. La identidad lógica de las funciones pedagógicas, la diversidad de las mismas y la significación del conocimiento de la educación exigen, en mi opinión, la puesta en marcha de las Facultades de Pedagogía, porque esta denominación es la única que define a los profesionales de la educación por su identidad lógica: el conocimiento de la educación específico que los capacita para explicar, interpretar y decidir la intervención pedagógica propia de la función para la que se habilitan.

En mi opinión, «Facultad de Pedagogía» es una denominación más adecuada que «Facultad de Educación» o «Facultad de Ciencias de la Educación».

En el primer caso, porque las facultades no se definen por el ámbito de conocimiento, que no es competencia exclusiva de ninguna institución, sino por el conocimiento del ámbito. Una Facultad de Educación, tendría como equivalente, aplicando el mismo principio, la facultad de los «dientes» (en lugar de estomatología) la de los «números» (en lugar de matemáticas), la de las «lenguas» (en lugar de Filología). Nadie está dispuesto a cometer este error en el caso de esas facultades; pero parece apropiado ir contra corriente y defender en el ámbito de la educación la denominación no por el conocimiento del ámbito (Pedagogía) sino por el ámbito de conocimiento (Educación).

En mi opinión, la denominación de Facultad de Educación es poco afortunada. No sólo por lo anteriormente dicho, sino porque se procede por mimetismo de lo anglo-sajón, sin pensar que el término «educación» tiene significado distinto en nuestro país. Lo paradójico del caso es que, cuando los anglosajones progresan y comienzan a hablar de la búsqueda del Pedagogo experto, y de la Pedagogía, nosotros retrocedemos e imitamos sus tradiciones de Facultad de Educación sin mucha imaginación.

En última instancia, la denominación de «Facultad de Educación» es un mal modo de identificar a los pedagogos — cualquiera que sea su función pedagógicaPor una parte, se apunta a la exclusividad del tema «educación» para los graduados de este centro (por la propia connotación del término Facultad) y esto es erróneo, porque la educación es una preocupación de muy diversos colectivos y ninguno puede patrimonializarla. Pero, además y, por otra parte, la denominación de Facultad de Educación supone la desaparición nominal de los pedagogos. Esto es así, porque no existiría de este modo la denominación «pedagogo» en ninguna titulación ni en ningún centro. El sentido del texto predica que de la Facultad de Medicina, son los médicos; de la Facultad de Psicología, son los psicólogos; de la Facultad de Sociología, son los sociólogos: ¿Por qué forzamos a predicar, fuera de la regla semántica, que de la Facultad de Educación, son los pedagogos? Esto es hacer un flaco servicio a la profesión. 
En mi opinión, entre Facultad de Pedagogía y Facultad de Educación no hay color ni duda a la hora de decidir. Y en este mismo sentido hay que definirse frente a la denominación de Facultad de Ciencias de la Educación. En este caso, si bien es verdad que se define la facultad por el conocimiento del ámbito, también lo es que la denominación «Ciencias de la Educación» responde a una concepción del conocimiento de la educación subalternada, que oculta la distinción entre estudios científicos de un ámbito y ciencia de ese ámbito (en nuestro caso, la Pedagogía como disciplina científica autónoma).

Adviértase que en esta polémica, el error más grave es tratar de obviarla, diciendo que es una disputa nominalista. Quien no se dé cuenta de que detrás de estas denominaciones están cuestiones técnicas rigurosas y problemas de profesionalismo irrefrenables por más tiempo, todavía no ha empezado a considerar seriamente el tema y las circunstancias sociológicas de la reforma de los títulos de enseñanza superior.

En el caso de la denominación Facultad de Ciencias de la Educación estamos manteniendo en el mismo plano de discusión dos cuestiones distintas, la identificación de un gremio académico y las materias que debe estudiar el gremio para formarse. Ningún gremio académico estudia sólo materias sustantivas de su ámbito (los filólogos no estudian solo filologías, ni los psicólogos estudian sólo psicologías, ni los médicos sólo disciplinas médicas); pero todos ellos definen su Centro por sus conocimientos sustantivos: la filología, la psicología, la medicina.

No caer en la cuenta de esta confusión entre identificación de gremio académico y materias de estudio nos llevaría al absurdo de defender en lugar de Filología, la Facultad de Ciencias de la lengua y el habla; en lugar de Psicología, Facultad de Ciencias del comportamiento, etc., etc., etc.

En el fondo, y hay que denunciarlo así, cuando se reduce la denominación del centro a Facultad de Ciencias de la Educación, estamos apoyando una denominación que no se defiende para otros casos semejantes y en igualdad de condiciones. La opción Ciencias de la Educación es favorecer una tendencia trasnochada ya en el ámbito de la investigación pedagógica: aquello que reduce el saber de educación a la teoría práctica, negando a la Pedagogía el estatus epistemológico de una estructura de conocimiento teórico consolidado, capaz de generar explicaciones autónomamente.

Frente a estas denominaciones, la Facultad de Pedagogía se define por el conocimiento del ámbito. Su cometido es dar formación en competencias pedagógicas. $\mathrm{Su}$ tarea no es, por tanto, la competencia en áreas culturales. Se entiende, por consiguiente, que lo fundamental es diseñar los créditos necesarios para capacitar a los especialistas con el conocimiento de la educación, sin perjuicio de que los créditos correspondientes a áreas culturales se cursen en otras facultades.

El modelo de Facultad de Pedagogía identifica profesionalmente a los especialistas en funciones pedagógicas y es un modelo en el que los planes de estudios establecen, racionalmente y de modo interno, relaciones entre carreras de especialización en función pedagógicas por medio de núcleos pedagógicos de formación de carácter parcialmente coincidente o compartido. Es posible hablar y construir Facultades de Pedagogía que integren administrativamente en un sólo centro los estudios 
conducentes a todas las titulaciones de las funciones pedagógicas, ya sean estas funciones de docencia, de apoyo al sistema educativo o funciones de investigación pedagógica.

\section{BIBLIOGRAFÍA}

BANCKS, O. (1983): Aspectos sociológicos de la educación. Narcea, Madrid.

BECKER, H. S. (1962): «The nature of a profession». En N. B. Henry (ed.); Education for the professions. National Society for the Study of Education. Chicago.

COMISIÓN (1986): «Documento de bases para la elaboración del estatuto del profesorado». Escuela Española (2.802, de 6 de febrero).

ESCOLANO, A. (1979): «Los estudios de ciencias de la educación: problemática curricular y profesional». En A. Escolano et al.: Los estudios de ciencias de la educación: currículum $y$ profesiones (11-40). ICE Universidad de Salamanca. Salamanca.

- (1980): «Diversificación de profesiones y actividades educativas». Revista Española de Pedagogía (147).

- (1982): «Las escuelas normales. Siglo y medio de perspectiva histórica». Revista de Educación. 30 (269).

FURTER, P. (1983): Les espaces de la formation. Presses Polytechniques Romandes, París.

GARCÍA CARRASCO, J. (1980): «Ciencias de la educación y profesiones pedagógicas. Problemas académicos y curriculares». VII Congreso Nacional de Pedagogía. Vol. I. S.E.P. Madrid.

- (1983): La ciencia de la educación. Pedagogos ¿para qué? Santillana. Madrid.

- (1984): Teoría de la Educación. Diccionario de Ciencias de la Educación. Anaya, Madrid.

- (1988): Apuntes de Teoría de la Educación. Secretaría de publicaciones de la Universidad de Salamanca, Salamanca.

GARCÍA GARRIDO, J. L. (1984): Sistemas educativos de hoy. Dykinson, Madrid.

GIMENO, J. (1982): «La integración de la teoría del aprendizaje en la teoría y práctica de la enseñanza». En A. Pérez Gómez y J. Almaraz: Lecturas de aprendizaje y enseñanza (467469). Zero, Madrid.

- (1982, a): «La formación del profesorado en la Universidad. Las escuelas universitarias de formación del profesorado de E.G.B.». Revista de Educación. 30 (269).

MEDINA RUBIO, R. (1980): «Expectativas socio-profesionales de las cualificaciones pedagógicas universitarias». VII Congreso Nacional de Pedagogía. Vol. I. S.E.P., Madrid.

ORDEN, A. de la (1979): «Enseñanza superior de Pedagogía y formación del profesorado». En A. Escolano et al.: Estudios de Ciencias de la Educación. Currículum y profesiones (243-252). ICE Universidad de Salamanca, Salamanca.

- (1984): «Investigación pedagógica experimental y praxis educativa». En Cincuentenario de los estudios universitarios de pedagogía (53-72). Facultad de Filosofía y Ciencias de la Educación, Madrid.

ORTEGA, J. (1980): «Estatuto socio-profesional del pedagogo». VII Congreso Nacional de Pedagogía. Vol. I. S.E.P., Madrid.

RUIZ BERRIO, J. (1979): «Antecedentes históricos de las actuales Secciones de Pedagogía». En A. Escolano et al.: Los estudios de ciencias de la educación: curriculum y profesiones (221-242), ICE Universidad de Salamanca, Salamanca. 
SANDERS, O., y MCPECK, J. (1976): «Theory into practice or viceversa. Comments on an educational antinomy». The Journal of Educational Thought. 10 (3).

SANVISENS, A. (1979): «La función del pedagogo en la sociedad actual». En A. Escolano et al.: «Los estudios de ciencias de la educación: currículum y profesiones (41-68). ICE de la Universidad de Salamanca, Salamanca.

- (1984): La formación de profesionales de la educación. Departamento de Pedagogía Sistemática de la Universidad de Barcelona, Barcelona.

- (1987): La Universidad española y las nuevas demandas científicas y sociales en el área de las ciencias de la educación. Universidad de Barcelona, P.P.U., Barcelona.

ToURIÑán, J. M. (1984): «La imagen social de la Pedagogía». Bordón (253), 600-630.

- (1987, a): Estatuto del profesorado. Función pedagógica y alternativas de formación. Escuela Española, Madrid.

- (1987, b): «Función pedagógica y profesionales de la educación». Bordón (266), 31-51.

- (1987, c): Teoría de la educación. Anaya, Madrid.

- (1988): «Formación del profesorado: consideraciones de base para una modificación de la propuesta del M.E.C.». Bordón. 40 (3), 467-78.

- (1989): «Profesionalización como principio del sistema educativo y función pedagógica». Revista de Ciencias de la Educación. (139).

- (coor.) (1990): «Formación técnico profesional y mercado de trabajo». Ponencia presentada al IX Seminario Interuniversitario de Teoría de la Educación. Departamento de Teoría e Historia de la Educación de la Universidad de Sevilla, Sevilla.

- (1991): «La profesionalización como principio del sistema educativo». Homenaje al profesor R. Marín. U.N.E.D., Madrid (en prensa).

VARIOS (1980): La investigación pedagógica y la formación de profesores. VII Congreso Nacional de Pedagogía. S.E.P., Madrid.

- (1984): Educación y Sociedad plural. (2 vol.). VII Congreso Nacional de Pedagogía. Secretaría de Publicaciones de la Universidad de Santiago y S.E.P., Santiago de Compostela.

- (1988): La calidad de los centros educativos. IX Congreso Nacional de Pedagogía. S.E.P., Alicante. 